\title{
As tecnologias de informação e comunicação na comunicação aumentativa e alternativa: um estudo quantitativo exploratório
}

\section{Information and communication technologies in augmentative and alternative communication: a quantitative exploratory study}

\author{
Patrícia Pinheiro, Anabela Cruz-Santos, Maria João Gomes \\ Instituto da Educação /CIEd - Universidade do Minho
}

\begin{abstract}
Resumo
As tecnologias de apoio podem ser um interface de comunicação para muitas crianças e jovens com necessidades educativas especiais. Nesta investigação, cuja abordagem metodológica é quantitativa e exploratória, recorremos ao inquérito por questionário como instrumento de recolha de dados. A amostra foi constituída por 85 professores especializados a desempenhar funções em unidades de educação especial. O objetivo deste estudo foi conhecer a perspetiva dos professores quanto à utilização, potencialidades e condicionantes das tecnologias na comunicação aumentativa e alternativa. Concluímos que os professores se preocupam em fazer formação regular na área das tecnologias. Conhecem a maioria das soluções tecnológicas apresentadas e utilizam tecnologia na sala de aula e tecnologias de apoio para a comunicação aumentativa e alternativa.

Palavras-chave: Comunicação aumentativa e alternativa, tecnologias de informação e comunicação.
\end{abstract}

\begin{abstract}
Assistive technologies can be a communication interface for many children and young people with special educational needs. In this research, whose methodological approach is quantitative and exploratory, we used the questionnaire survey as data collection instrument. The sample consisted of 85 specialized teachers working in special education units. The objective of this study was to know the teachers' perspective regarding the use, potentialities and conditioning of technologies in augmentative and alternative communication. We conclude that teachers are concerned with regular training in the area of technology. They know most of the technological solutions presented and use technology in the classroom and technologies of support for augmentative and alternative communication.

Keywords: Alternative and augmentative communication, information and communication technologies.
\end{abstract}

\section{Introdução}

A escola tem um papel central na educação e na preparação de todos os alunos em termos do futuro, incluindo daqueles com Necessidades Educativas Especiais (NEE).
A inclusão é fundamental para que todos os alunos "tenham direito a uma educação de qualidade, para que todos os alunos sejam vistos no seu todo quanto ao crescimento e desenvolvimento" (Nielsen, 1999, p.9). Garantir a inclusão é um dever coletivo e um direito a adquirir (Santos, 2006; Correia, 2005).

Ao nível das crianças e jovens com NEE um dos maiores desafios que se colocam relativamente à sua inclusão escolar e social é assegurar adequadas condições de comunicação, nos casos em que a mesma se encontra comprometida ou diminuída. A procura das melhores soluções para os problemas de comunicação que afetam muitas das crianças com NEE passa pelo conhecimento das alternativas existentes, nomeadamente aquelas que fazem uso das tecnologias digitais. Nesse sentido, importa conhecer as perceções, práticas e nível de formação que os professores especializados a desempenhar funções em unidades de Educação Especial do sistema educativo português possuem relativamente a esta temática. Tendo presente este objetivo geral, levou-se a cabo um estudo de tipo survey com base num questionário dirigido aos professores especializados a desempenhar funções em unidades de Educação Especial no ano letivo de 2013/2014. Este texto visa apresentar os principais dados e conclusões decorrentes deste estudo, no sentido de permitir caracterizar a situação existente.

\section{Metodologia}

Nas ciências sociais encontramos uma grande variedade de estudos descritivos classificados sob a denominação de Inquérito. Coutinho (2011) refere que constituem o plano de investigação mais utilizado em investigação social. Os inquéritos contribuíram significativamente para o conhecimento que se tem hoje dos contextos sociais, sendo um dos métodos utilizados com mais frequência em trabalhos de investigação no âmbito das ciências sociais. Existem diferentes tipos de Inquérito que suscitam um conjunto de questões teóricas, epistemológicas e metodológicas.

Coutinho (2011, p. 276) refere que "os procedimentos de investigação por Inquérito (...) devem estabelecer regras que nos permitam aceder de forma científica ao 
que os inquiridos opinam, o que na prática equivale a dizer que um segundo investigador possa repetir todo o processo".

O seu objetivo fundamental é, portanto, a descrição das caraterísticas de determinada população e/ou fenómeno ou o estabelecimento de relações entre variáveis. Procura verificar, com a precisão possível, a frequência com que um fenómeno ocorre, a sua natureza, as suas caraterísticas e a sua relação com os outros.

Pinsonneault e Kraemer (1993), referidos em Freitas et. al. (2000), classificam o Inquérito quanto à sua intenção, nomeadamente: explanatória (testar uma teoria e as relações causais), exploratória (familiarizar-se com um tema ou identificar os conceitos iniciais, dar ênfase na determinação de quais conceitos devem ser medidos e como devem ser medidos, descobrir novas possibilidades e dimensões da população de interesse) e descritiva (quais situações, eventos, atitudes ou opiniões que estão manifestadas numa população; descreve a distribuição de algum fenómeno na população ou entre os subgrupos da população ou, ainda, faz uma comparação entre essas distribuições).

Tendo em consideração o exposto, podemos inserir o estudo desenvolvido nos Planos Não-Experimentais ou Descritivos especificamente na tipologia Inquérito (Coutinho, 2011, p. 276), como já referimos anteriormente. Entendemos que uma metodologia de tipo descritivo foi a mais adequada para realizar esta investigação, já que segundo Fink (1995) trata-se de um método de recolha de informação que permite descrever, comparar, ou explicar conhecimentos, atitudes e comportamentos.

Portanto o nosso estudo constituiu-se como um estudo descritivo de caráter quantitativo, desenvolvido sob a forma de um inquérito por questionário, cujos dados foram analisados de através de análise estatística.

\section{Questões de investigação}

O estudo que apresentamos teve como objetivo principal investigar o uso das TIC na CAA tendo como foco central os conhecimentos, as práticas e as perceções dos professores especializados a desempenhar funções no ano letivo de 2013/2014 nas Unidades de Ensino Estruturado para a Educação de Alunos com Perturbações do Espectro do Autismo (UEEA) e nas Unidades de Apoio Especializado para a Educação a Alunos com Multideficiência e Surdocegueira Congénita (UAEM) do sistema educativo português.

A opção pela inclusão no estudo dos professores especializados a desempenhar funções nestas unidades decorreu do facto das UEEA e das UAEM constituírem uma resposta educativa especializada desenvolvida em escolas ou agrupamentos de escolas que concentrem grupos de alunos que manifestem perturbações enquadráveis nestas problemáticas, sendo que estes alunos são na generalidade os que mais necessitam de SCAA.

Decorrentes do objetivo principal atrás referido definimos um conjunto de objetivos mais específicos, que nos permitiram operacionalizar o processo de recolha e análise dos dados:
- Analisar se os professores que atuam nas UAEM e nas UEEA conhecem e utilizam SCAA e SCAA em suporte tecnológico.

- Analisar as perspetivas dos professores que atuam nas UAEM e nas UEEA relativamente ao potencial do uso das TIC no suporte à CAA.

- Analisar as perceções dos professores que atuam nas UAEM e nas UEEA relativamente aos fatores condicionantes do uso das TIC no suporte à CAA.

\section{Amostra}

A amostra é de natureza probabilística, uma vez que o universo de inquirição eram todos os professores a desempenhar funções nas UAEM e UEEA, sendo que, teoricamente, qualquer membro dessa população poderia ter integrado o conjunto de professores respondentes que veio a constituir a amostra. A principal razão desta escolha prende-se com o potencial das unidades de Educação Especial na utilização das TIC na CAA, considerando as dificuldades e limitações ao nível da comunicação da população atendida nestes contextos.

A amostra reuniu professores de todas as Direções Gerais dos Estabelecimentos Escolares, embora com mais representatividade a DGEstE Norte, seguindo-se a DGEstE Lisboa e Vale do Tejo, sendo constituída por um total de 85 elementos, maioritariamente do sexo feminino.

Metade dos sujeitos que constituíram a amostra tem até 10 anos de serviço, a restante tem entre 11 a 35 anos de serviço. A categoria profissional que prevalece é Professor de Quadro de Agrupamento/ Escola, embora a categoria Professor contratado esteja quase ao mesmo nível no que se refere ao número de inquiridos. A licenciatura pré Bolonha é a habilitação académica dominante. Relativamente aos contextos de atuação, a tipologia UAEM é a que reúne o maior número de respostas, o que era expectável uma vez que o número destas unidades na rede de apoio à Educação Especial é também mais elevado.

\section{Instrumento de recolha de dados}

$\mathrm{O}$ estudo que desenvolvemos assumiu um caráter exploratório e descritivo, adotando uma abordagem de survey de carácter quantitativo.

$\mathrm{Na}$ recolha de dados foi utilizado como instrumento o inquérito por questionário eletrónico distribuído a nível nacional. Para tal recorremos à rede de estabelecimentos escolares, cujos contactos estavam disponíveis na Direção Geral dos Estabelecimentos Escolares (DGEstE). O inquérito por questionário foi devidamente autorizado pela entidade de Monitorização de Inquéritos em Meio Escolar (MIME). Os dados recolhidos foram alvo de tratamento estatístico através do Statistical Package for Social Sciences (SPSS v.22).

\section{Procedimento de recolha e tratamento de dados}

O questionário foi construído especificamente para este estudo e foi implementado e difundido através da ferramenta online Survey Monkey.

Os dados foram recolhidos junto dos professores que no ano letivo de 2013/2014 se encontravam a lecionar nas UAEM e UEEA a nível nacional continental. 
A aplicação do questionário foi devidamente autorizada pela entidade responsável pela utilização de instrumentos de inquirição em meio escolar (Monitorização de Inquéritos em Meio Escolar - MIME), de acordo com os normativos em vigor. A respetiva autorização integra os anexos deste projeto.

Optamos por implementar o questionário em formato digital por razões de economia financeira e de tempo, pois evitou custos de reprodução dos questionários, de envio e de deslocações, que seriam incomportáveis já que o mesmo foi divulgado a nível nacional

Devido a causas exógenas foi-nos exigido um esforço maior na divulgação do mesmo junto das respetivas Direções de Serviços Regionais da Direção Geral dos Estabelecimentos Escolares (DGEstE) e das Direções dos Agrupamentos de Escolas. Para se conseguir uma maior divulgação do questionário e consequentemente o seu preenchimento por professores distribuídos pelas diversas regiões, foram contatadas as entidades descritas anteriormente por correio eletrónico consoante os endereços que constavam na rede de contactos da DGEstE. Muitos endereços eletrónicos encontravam-se desatualizados, pelo que foram devolvidos inúmeros e-mails ao remetente por falha de envio. Foi portanto necessário a atualização exaustiva dos mesmos recorrendo ao cruzamento de dados da rede de contatos disponível na DGEstE e da Direção-Geral da Educação (DGE) e ainda consultando os sítios dos Agrupamentos em falta.

$\mathrm{Na}$ mensagem de correio eletrónico, na qual se solicitava aos Diretores dos agrupamentos de escolas o reencaminhamento para os professores a desempenharem funções nas unidades de Educação Especial, incluía-se também a hiperligação de acesso ao questionário, bem como uma nota informativa e explicativa de garantia de anonimato de acordo com a Lei n. ${ }^{\circ} 67 / 98$ de 26 de outubro.

Tivemos um retorno de aproximadamente 200 questionários, dos quais 154 completamente preenchidos. Todavia, apenas consideramos para o nosso estudo 85 questionários que preenchiam um requisito fundamental e devidamente especificado - professores especializados a desempenhar funções nas UAEM e UEEA no ano letivo 2013/2014. Tivemos diversos contactos de terapeutas e professores que atuavam com alunos com NEE utilizadores de SCAA embora noutros contextos não solicitados no nosso estudo, que gostariam de conhecer os resultados da inquirição e também poder preencher o questionário. Para o sucedido podemos elencar algumas possíveis explicações: os Diretores reencaminharem o email para todos os docentes de Educação Especial do Agrupamento, os outros profissionais (professores e terapeutas) a desempenhar funções noutros contextos tinham intenção de participar e gostariam de contribuir para a nossa inquirição, entre outras. Porém, também recebemos de alguns diretores respostas negativas à nossa solicitação de divulgação

Os dados recolhidos foram processados em software de tratamento estatístico de dados (SPSS v.22) de acordo com as indicações da bibliografia, nomeadamente a análise de cariz quantitativo.

\section{Resultados}

Dada a extensão do questionário utilizado, envolvendo 22 questões, e considerando as limitações de dimensão deste texto optamos por apresentar apenas as principais constatações e conclusões decorrentes do estudo, tendo em conta os objetivos do mesmo já apresentados.

\section{Utilização das TIC, conhecimentos e formação em TIC na educação especial e utilização}

Embora os professores inquiridos revelem utilizar diariamente as TIC, quer em contexto pessoal e familiar, quer no contexto profissional, quase metade $(43,5 \%)$ dos inquiridos respondeu não ter tido qualquer formação em TIC no curso de formação inicial.

No que se refere à formação em TIC direcionada para a Educação Especial e para a CAA recolhemos dados que nos causaram inquietação, uma vez que a percentagem de inquiridos que responderam afirmativamente é muito reduzida $(17,6 \%)$

Quanto à formação do domínio da Educação Especial, todos os professores inquiridos possuem formação especializada, através de curso de formação especializada ou mestrado em Educação Especial.

Concluímos ainda que os professores inquiridos procuram formação através de diversas fontes: meios formais (cursos superiores, formação contínua, etc) e meios informais (auto-estudo, contato com colegas, etc.), o que nos revela preocupação por uma constante atualização científica e pedagógica.

A principal razão que levou os professores a procurar formação além da formação inicial foi "aprendizagem de novas metodologias de trabalho e intervenção". A opção "superação de défices de formação inicial" foi uma das menos selecionadas, o que nos causa surpresa já que a quase totalidade os professores referiram que não teve formação direcionada para as TIC e para as CAA no curso de formação inicial e expectávamos que essa necessidade fosse mais premente, mas que pode já ter sido compensada através da formação contínua formal e informal. Relembramos também que se solicitou aos professores que indicassem apenas a "principal razão".

\section{Conhecimentos específicos no campo dos SCAA}

Entrando num domínio mais específico, perante uma listagem de SCAA (signos gráficos, Makaton, PIC, Sistema BLISS, SPC, Sistema Rebus e Sistema de símbolos para alfabetização da Widgit) os professores manifestaram conhecer e utilizar a generalidade, à exceção do Sistema Rebus e do Sistema de símbolos para a alfabetização da Widgit. Era nossa expectativa que a percentagem de conhecimentos e utilização fosse mais evidente e significativa em alguns SCAA, uma vez que estamos a lidar com um grupo de professores especializados na área da educação Especial e a trabalhar em contextos muito específicos e nos quais a possibilidade de uso de SCAA é maior.

De uma forma geral todos as tecnologias de apoio ao nível de hardware para a CAA elencados no questionário são do conhecimento dos inquiridos e o seu grau de utilização é bastante satisfatório. 
Quanto às tecnologias de apoio ao nível de software para a CAA fizemos a separação entre os de acesso livre, os quais se podem descarregar da web, e os homologados e comercializados pelos agentes nacionais. No que respeita ao conhecimento e utilização pelos professores inquiridos, as tecnologias de apoio ao nível de software para a CAA de acesso livre apresentam diferenças muito significativas comparativamente com as tecnologias de apoio para a CAA de caráter comercial.

\section{Nível de utilização de SCAA e de SCAA em suporte TIC}

Compreender o nível de utilização de SCAA e de SCAA em suporte TIC era também um propósito. Assim, fizemos um levantamento do número professores que trabalham em unidades de Educação Especial cujos alunos que utilizam SCAA com e sem suporte TIC. O mais relevante a destacar é que 66 dos 85 professores que constituíram a nossa amostra trabalham em unidades de Educação Especial em que há utilização de "SCAA em suporte TIC".

A utilização pelos alunos de SCAA com e sem recurso às TIC nas atividades de aprendizagem, socialização e autonomia nos diversos contextos quotidianos é evidente. $\mathrm{O}$ contexto em que os alunos mais utilizam os SCAA com e sem recurso às TIC é na unidade de Educação Especial, sucedido pela escola/ aulas/ turma e por fim no contexto familiar. Assim, constata-se que o nível de utilização é maior nos contextos que envolvem a presença com professores especializados. A utilização de SCAA com e sem recurso às TIC vai diminuindo à medida que o contexto deixa de estar sob a supervisão dos mesmos. A utilização de SCAA em dispositivos móveis não é tão acentuada como a utilização de SCAA ou a utilização de SCAA em suporte TIC e representa aproximadamente metade do nível de utilização de SCAA em suporte TIC. O mesmo se reflete na escola/ aulas/ turma e no contexto familiar. Quando o nível tecnológico aumenta, o nível de utilização diminui. E quando o contexto de utilização se afasta do contexto mais restrito das unidades de Educação Especial e se aproxima do contexto familiar também diminui o nível de utilização.

Perspetivas dos professores que atuam nas UAEM e nas UEEA relativamente ao potencial do uso das TIC no suporte à CAA

Para dar resposta ao segundo objetivo da nossa investigação: analisar as perspetivas dos professores que atuam nas UAEM e nas UEEA relativamente ao potencial do uso das TIC no suporte à CAA, os professores foram solicitados no sentido de exporem o seu grau de concordância perante quatro afirmações relacionadas com a conjunção TIC e CAA.

Considerando a experiência profissional dos professores inquiridos concluímos que atribuem um grau de concordância elevado com as afirmações elencadas. São unânimes na importância das TIC associadas à CAA enquanto facilitadoras do processo de socialização, autonomia e aprendizagem dos alunos com NEE, opinião coincidente com a literatura (Amante, 1993; Batanero, 2004; Suárez, Aguilar, Rosell, \& Basil, 2000; Rodrigues
\& Teixeira, 2006; Santos, 2006; Moreira, Ferreira \& Almeida, 2009). Concordam que os dispositivos móveis (ex. ipad, tablet, telemóvel) com SCAA integrados tornam os seus utilizadores mais independentes. Evidenciam também a influência positiva das TIC na implementação de SCAA e que os SCAA com recurso às TIC são mais eficazes do que os SCAA sem recurso às TIC.

Perceções dos professores quanto aos fatores condicionantes do uso das TIC no suporte à CAA

Relativamente ao terceiro objetivo da nossa investigação: analisar as perceções dos professores que atuam nas UAEM e nas UEEA relativamente aos fatores condicionantes do uso das TIC no suporte à CAA retiramos as seguintes conclusões baseadas na experiência profissional dos professores inquiridos, que mais uma vez a literatura corrobora (Amaral et al, 2004; Correia, 1999; Faias, 2012; Resolução do Conselho de Ministros n. ${ }^{\circ}$ 110/2003; UNESCO, 2011).

Concluímos assim que os professores atribuem grande importância aos conhecimentos dos técnicos especializados quanto aos produtos de apoio. Atribuem também, e de forma expressiva, importância à formação específica dos professores e outros técnicos que atuam nas unidades de Educação Especial relativamente aos SCAA com recurso às TIC. Referem que a morosidade do processo de avaliação e atribuição dos produtos de apoio/ajudas técnicas/ tecnologias de apoio é um fator de muita relevância no que se refere ao sucesso da implementação de SCAA. De igual modo, o longo período de aprendizagem dos alunos relativamente ao uso de SCAA com base nas TIC e a inexistência de SCAA com base nas TIC adequados a certas problemáticas são fatores que os professores consideram muito importantes. A falta de recursos nas unidades de Educação Especial durante os períodos de espera pelos produtos de apoio prescritos aos alunos e a disponibilidade das famílias para a implementação em contexto familiar de SCAA com recurso às TIC são também fatores de muita relevância no sucesso da implementação das SCAA em suporte TIC.

\section{Conclusões}

Permitir a todas as pessoas o acesso à informação e ao conhecimento, criando as condições necessárias para a aquisição de competências no domínio das TIC, constitui, atualmente, um imperativo inadiável para qualquer sociedade que não queira ver-se irremediavelmente ultrapassada no percurso imparável da globalização e da predominância crescente daquelas tecnologias em todos os sectores da vida humana.

Cada nova tecnologia traz em si um elevado poder de inclusão ou exclusão das pessoas no seu meio. As TIC permitem ultrapassar barreiras, quer através de produtos de apoio mais sofisticados, quer pelas potencialidades dos novos produtos e serviços do mercado.

A nosso ver, a TIC são de facto essenciais para a inclusão das crianças com NEE, constituem uma ferramenta valiosíssima para a comunicação, autonomia e aprendizagem pessoais e sociais. Contudo, as TIC por 
si só não colmatam todas as limitações ou evidenciam todas as capacidades. A sua implementação e efetiva utilização carece de uma formação prévia e constante dos profissionais técnicos envolvidos entrosados com as famílias. Carece ainda de um trabalho de gestão de expetativas quer dos profissionais, das famílias e dos seus utilizadores, uma vez que é um caminho marcado por avanços e recuos. Considerando a nossa experiência profissional, para o sucesso da implementação de um SCAA é necessário verificar o potencial e aptidões do utilizador, estabelecer etapas curtas e ser paciente, pois os resultados podem ser morosos.

Os SCAA em suporte TIC são mais uma possibilidade e em alguns casos podem ser a melhor solução, noutros casos podem ser a única solução e em outros casos podem não ser solução alguma. O que queremos realçar é que existindo mais esta opção, ela deve ser conhecida e utilizada quando adequada.

\section{Referências Bibliográficas}

Amaral, I. (2002). Characteristics of communicative interactions between children with multiple disabilities and their non-trained teachers: Effects of an intervention process. Tese de doutoramento. Porto: Faculdade de Psicologia e Ciências da Educação da Universidade do Porto.

Correia, L. M. (1999). Alunos com necessidades educativas especiais nas classes regulares. Porto: Porto Editora.

Correia, L. M. (2005). Inclusão e necessidades educativas especiais: Um guia para educadores $e$ professores. Porto: Porto Editora.

Faias, J. (2012). Tecnologias de apoio - prestação de serviços. Porto: Instituto Politécnico do Porto - Escola Superior de Tecnologia da Saúde do Porto.

Freixo, A. R. (2013). A importância da comunicação aumentativa/alternativa em alunos com paralisia cerebral no $1^{\circ}$ ciclo do ensino básico. Tese de dissertação de mestrado. Lisboa: Escola Superior de Educação João de Deus.

Fontes, G., Abreu, S. (2010). WAACT - Widget Augmentative and Alternative Communication Toolkit. INForum 2010 - II Simpósio de Informática - 9-10 setembro, 353-364. Braga: Universidade do Minho.

Hanline, M., Nunes, M. \& Worthy B. (2007). Augmentative and Alternative Communication in the Early Childhood Years. Beyond the Journal: Young Children on the Web, 1-6.

Millar, S. \& Scott, J. (1998). What is Augmentative and Alternative Communication? An Introduction, 3-12. Acedido a 19 de dezembro de 2012 em de http://www.acipscotland.org.uk/Millar-Scott.pdf

Nielsen, L. B. (1999). Necessidades educativas especiais na sala de aula: Um guia para professores. Porto: Porto Editora.

Nunes, C. (2003). Crianças com multideficiência sem linguagem oral expressiva: Formas de comunicação mais utilizadas para fazer pedidos. Tese de dissertação de mestrado. Lisboa: Universidade Católica Portuguesa.

UNESCO - European Agency for Development in Special Needs Education (2011). ICTs in Education for people with disabilities - Review of innovative practice. Moscovo.

Ribeiro, J. M., Moreira, A. \& Almeida, A. M. (2009). Preparing special education frontline professionals for a new teaching experience. eLearning Papers, 16. Acedido a 10 de novembro de 2013 em http://www.openeducationeuropa.eu/en/article/Preparin g-special-education-frontline-professionals-for-a-new-te aching-experience.

Rodrigues, C., \& Teixeira, R. (2006). Tecnologias em processos de inclusão. Revista Faculdade de Educação, 31(2), p. 261-276. Goiás: Universidade Federal.

Rosell, C., \& Basil, C. (1998). Sistemas de signos manuales y sistemas de signos gráficos: características y criterios para su uso. Sistemas de signos y ayudas técnicas para la comunicación aumentativa y la escritura: princípios teóricos e aplicaciones. Barcelona: MASSON.

Santos, L. (2006). A escrita e as TIC em crianças com dificuldades de aprendizagem: Um ponto de encontro. Tese de dissertação de mestrado. Braga: Universidade do Minho.

Silva, M. O. E. da (2009). Da Exclusão à inclusão: Concepções e práticas. Revista Lusófona de Educação, (13), p.135-153. Recuperado em 07 de janeiro de 2015, de

http://www.scielo.gpeari.mctes.pt/scielo.php?script=sci _arttext\&pid=S1645-72502009000100009\&lng=pt\&tln $\mathrm{g}=\mathrm{pt}$.

Tetzchner, S., \& Martinsen, H. (2002). Introdução à comunicação aumentativa e alternativa. Porto: Porto Editora.

Decreto-Lei n. ${ }^{\circ}$ 3/2008 de 7 de janeiro. Ministério da Educação e Ciência. Lisboa.

Decreto-Lei n. ${ }^{\circ}$ 93/2009 de 16 de abril. Ministério do Trabalho e da Solidariedade Social. Lisboa.

Resolução do Conselho de Ministros n. ${ }^{\circ}$ 110/2003 de 20 de novembro. 